DESY 01-166

October 2001

\title{
Separating perturbative and non-perturbative contributions to the plaquette*
}

\author{
R. Horsley ${ }^{\mathrm{a}}$, P. E. L. Rakow ${ }^{\mathrm{b}}$ and G. Schierholz ${ }^{\mathrm{a}, \mathrm{c}}$

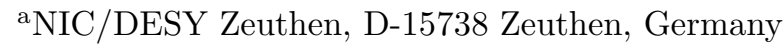 \\ ${ }^{\mathrm{b}}$ Institut für theoretische Physik, Universität Regensburg, D-93040 Regensburg, Germany \\ ${ }^{c}$ Deutsches Elektronen-Synchrotron DESY, D-22603 Hamburg, Germany
}

We try to separate the perturbative and non-perturbative contributions to the plaquette of pure $S U(3)$ gauge theory. To do this we look at the large- $n$ asymptotic behaviour of the perturbation series in order to estimate the contribution of the as-yet uncalculated terms in the series. We find no evidence for the previously reported $\Lambda^{2}$ contribution to the gluon condensate. Attempting to determine the conventional $\Lambda^{4}$ condensate gives a value $\sim 0.03(2) \mathrm{GeV}^{4}$, in reasonable agreement with sum rule estimates, though with very large uncertainties.

\section{INTRODUCTION}

There is a long history of trying to extract a non-perturbative gluon condensate from lattice calculations of the plaquette [1]. Thanks to the work of [2] we have a much longer perturbation series for the plaquette than for any other lattice quantity. On the other hand, the plaquette is one of the most ultra-violet dominated quantities, which means that any non-perturbative contribution is likely to be very small compared with the perturbative part.

Conventionally one expects that the nonperturbative part of the plaquette is proportional to $a^{4}$, and related to the gluon condensate introduced in [3]

$P_{M C}=P_{p e r t}-a^{4} \frac{\pi^{2}}{6^{2}}\left[\frac{-b_{0} g^{3}}{\beta(g)}\right]\left\langle\frac{\alpha}{\pi} G G\right\rangle$

where $b_{0}$ is the first coefficient of the $\beta$ function. However one study has reported a nonperturbative contribution scaling like $a^{2}$ 沟.

The Monte Carlo quenched plaquette, $P_{M C}$, can be measured very accurately. The difficult part in determining the condensate is finding the sum of the perturbative series $P_{\text {pert }} \equiv$ $1-\sum_{n} p_{n} g^{2 n}$. (We use $g^{2}$ as our expansion parameter, rather than $\beta^{-1}$.) We know the first few terms in this series from conventional lat-

\footnotetext{
*Talk given by P. Rakow at Lattice 2001, Berlin.
}

tice perturbation theory calculations. Di Renzo et al. have managed to calculate many more terms in the series by a clever stochastic method [2]. Since this is the only lattice quantity with a long perturbation series, it gives us a unique opportunity to see how the coefficients behave at large $n$. The calculations, which were done on a $24^{4}$ lattice, reproduced the three known terms correctly.

\section{EXTRAPOLATING THE SERIES}

To estimate any non-perturbative contributions to the plaquette, we need to know the sum of the perturbation series to one part in $10^{3}$ or better. We quickly see that for interesting couplings $\left(g^{2} \approx 1\right) 10$ loops is not enough, and that we need a good estimate of the contribution from higher order terms.

To extrapolate to higher loops, we need to know how the coefficients depend on $n$. A good way of determining this behaviour is to look at $r_{n}$, the ratio of adjacent coefficients. In statistical mechanics, $r_{n}$ is often plotted against $1 / n$ rather than $n$. This is because this gives a straight line for a series with a power-law behaviour,

$$
\begin{aligned}
& (1-u x)^{q}= \\
& \quad 1-q u x+\cdots+\frac{\Gamma(n-q)}{\Gamma(n+1) \Gamma(-q)}(u x)^{n}+\cdots
\end{aligned}
$$

so that 


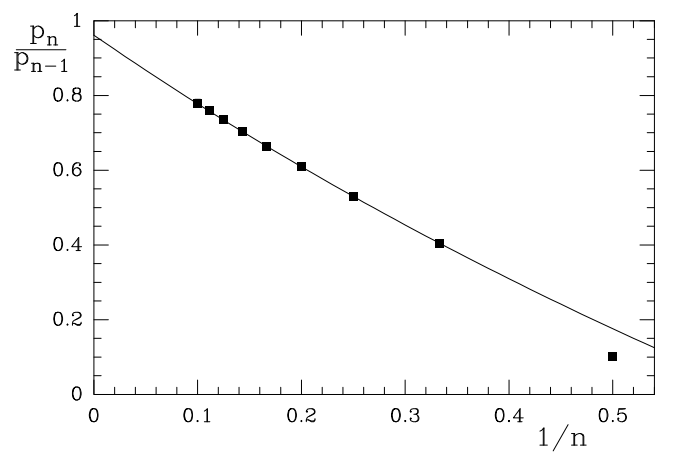

Figure 1. The ratio of successive coefficients in the perturbation series of the plaquette, plotted against $1 / n$. The solid line is the fit of eq. (4).

$c_{n} / c_{n-1}=u[1-(1+q) / n]$

where the $c_{n}$ are the coefficients in the Taylor expansion.

The plot, Fig. 11, is almost linear, though there is a little curvature, which is taken into account by making a fit of the form

$r_{n} \equiv \frac{p_{n}}{p_{n-1}}=u\left(1-\frac{1+q}{n+s}\right)$,

with $u=0.961(9), q=0.99(7), s=0.44(10)$. This series converges for $\left|g^{2}\right|<|u|^{-1}$. At first sight it might seem surprising that a series with a power-law singularity describes the data well. However, it is known that the specific heat of lattice gauge theories has a sharp peak in the crossover region between strong and weak coupling (for an example, see [5]). If this feature dominates the series, a fit like eq.(4) is reasonable.

To estimate the higher-order terms in the series, [4] use a different extrapolation, based on renormalon ideas about the asymptotic form of the series. Their formula for the coefficients is quite complicated, for full detail, see [4]. They assume that there is a scheme in which renormalon behaviour (factorial growth of the coefficients) applies, and that the coupling in this scheme is related to the usual lattice coupling by a transformation of the type

$\frac{1}{g_{\text {ren }}^{2}}=\frac{1}{g^{2}}-\frac{r}{6}-\frac{r^{\prime}}{6^{2}} g^{2}$.

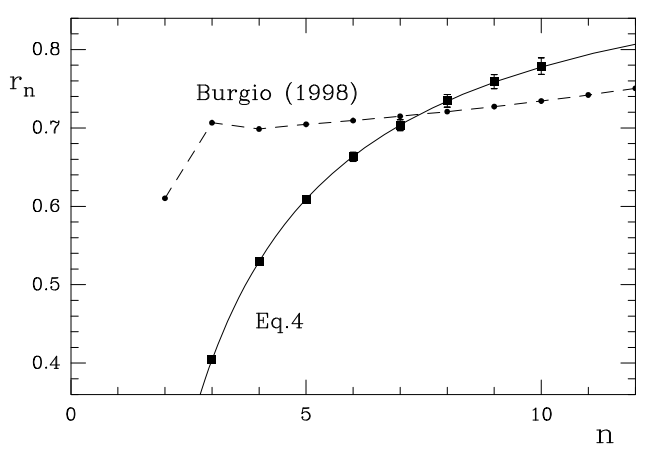

Figure 2. The ratio $r_{n}$ compared with the renormalon fit of [4] (dashed line) and the fit of eq.(值) (solid line).

Using the coefficients known at the time (up to $n=8$ ) Burgio et al. made a fit for $r$ and $r^{\prime}$ which leads to the dashed line shown in Fig. 2. Although this renormalon fit has about the right value near $n \approx 8$, it does not reproduce the $n$-dependence as well as the fit eq.(伯).

The resulting fit for the $p_{n}$ is shown in Fig. 3, and compared with the renormalon-inspired fit. Eq.(1) describes the coefficients from 2 loops onward very well, while the renormalon fit shows much less curvature than the data. The new extrapolation predicts larger coefficients for the unknown terms in the perturbation series. That means that there will be a smaller condensate left after subtracting $P_{\text {pert }}$.

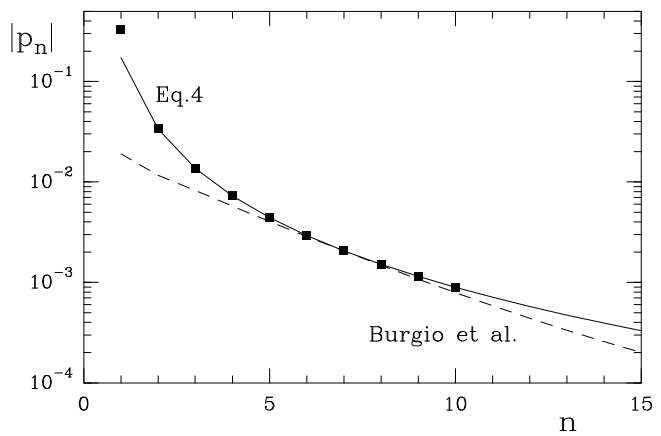

Figure 3. The coefficients in the plaquette series, compared with the fit of 国 (dashed line) and of eq.(国) (solid line). 


\section{THE GLUON CONDENSATE}

Using the 8 coefficients which they had then, and estimating the remainder of the perturbative series from their renormalon fit, [4] produced values for the non-perturbative part of the plaquette for $\beta$ between 6.0 and 7.0. The surprising result was that $\Delta P$, the difference between the perturbative plaquette and the Monte Carlo result, was not proportional to $a^{4} \Lambda^{4}$ as conventionally expected, but to $a^{2} \Lambda^{2}$.

In Fig. A we compare $\Delta P$ using our estimate of the perturbative plaquette, and using the esti-

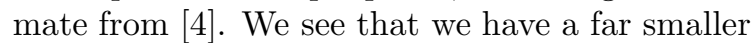
condensate left over, and that there is no longer much support for the unconventional $a^{2}$ slope.

In Fig. 5 we see that $\Delta P$ scales roughly as $a^{4}$, as expected from eq.(11). However the errors (mainly from the uncertainty in summing the perturbation series) are very large. The dashed lines show the values of the gluon condensate expected according to various sum-rule estimates [3,6]. Using the force scale $r_{0}$ to set our length scale gives

$$
\left\langle\frac{\alpha}{\pi} G G\right\rangle \sim 0.03(2) \mathrm{GeV}^{4},
$$

which is similar to phenomenological estimates such as $\sim 0.012 \mathrm{GeV}^{4}$ [3] or $0.024(8) \mathrm{GeV}^{4}$ [6].

\section{CONCLUSIONS}

We can see from Figs 1 - 3 that the power law fit, eq.(4), describes the known perturbative co-

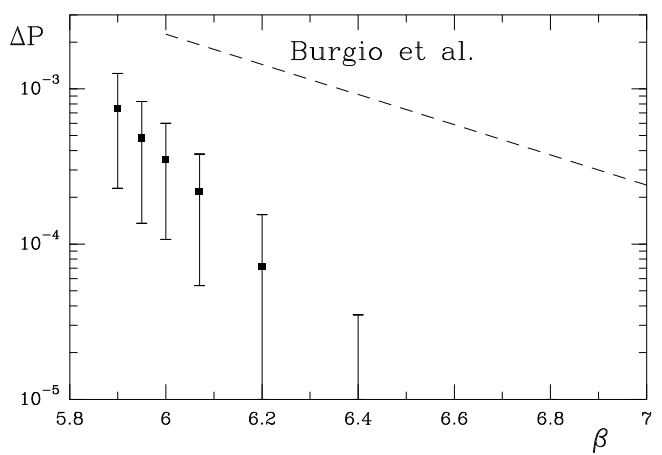

Figure 4. $\Delta P$, the difference between the Monte Carlo and perturbative plaquette, according to this work (points), and according to (dashed line).

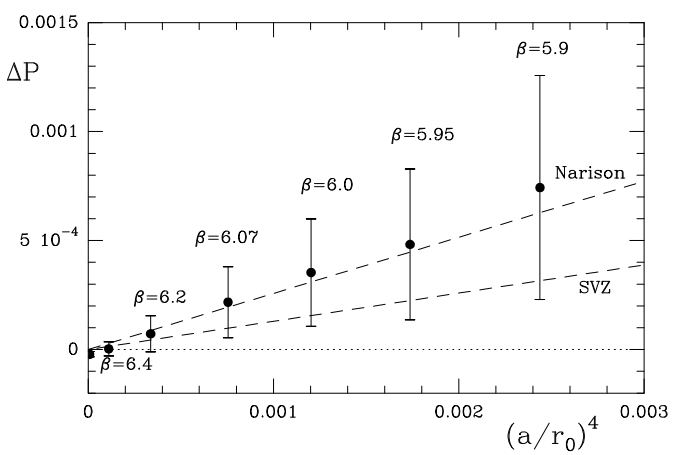

Figure 5. $\Delta P$, compared with phenomenological values of the gluon condensate from [3, 60.

efficients better than the renormalon fit [4].

If we use eq.(4) to estimate the sum of the perturbative series, we find a number very close to the full plaquette as measured in Monte Carlo calculations. So any non-perturbative contribution to the plaquette is very small.

The non-perturbative contribution to the plaquette does not look proportional to $a^{2} \Lambda^{2}$, but rather appears to be proportional to $a^{4} \Lambda^{4}$, with about the same order of magnitude as the phenomenological gluon condensate.

Asymptotically, the renormalon behaviour could still be right. The renormalon asymptote predicts $r_{n} \approx n b_{0} / 2$, which does not catch up with eq.(位) until $n \approx 25$.

Acknowledgements: This work was supported by the DFG and the BMBF.

\section{REFERENCES}

1. T. Banks, R. Horsley, H. R. Rubinstein and U. Wolff, Nucl. Phys. B 190 (1981) 692; A. Di Giacomo and G. C. Rossi, Phys. Lett. B 100 (1981) 481.

2. F. Di Renzo and L. Scorzato, heplat/0011067.

3. M. A. Shifman, A. I. Vainshtein and V. I. Zakharov, Nucl. Phys. B 147 (1979) 385; 448.

4. G. Burgio, F. Di Renzo, G. Marchesini and E. Onofri, Phys. Lett. B 422 (1998) 219.

5. B. Lautrup and M. Nauenberg, Phys. Rev. Lett. 45 (1980) 1755.

6. S. Narison, Phys. Lett. B 387 (1996) 162. 